\title{
WEIGHTED ADDITIVE INFORMATION MEASURES
}

\author{
WOLFGANG SANDER \\ Institute for Analysis \\ University of Braunschweig \\ Pockelsstr. 14, D 3300 Braunschweig, Germany \\ (Received January 26, 1989 and in revised form May 25, 1990)
}

ABSTRACT. We determine all measurable functions $I, G, L:[0,1] \rightarrow \mathbf{R}$ satisfying the functional equation

$\sum_{i=1}^{n} \sum_{j=1}^{m} I\left(p_{i} q_{j}\right)=\sum_{i=1}^{n} \sum_{j=1}^{m} G\left(p_{i}\right) I\left(q_{j}\right)+\sum_{i=1}^{n} \sum_{j=1}^{m} L\left(q_{j}\right) I\left(p_{i}\right)$

for $P \varepsilon \Gamma_{n}, Q \in \Gamma_{m}$ and for a fixed pair $(n, m), n \geqslant 3, m \geqslant 3$, where $\mathrm{G}(0)=\mathrm{L}(0)=0$ and $\mathrm{G}(1)=\mathrm{L}(1)=1$. This functional equation has interesting applications in information theory.

KEY WORDS AND PHRASES. Weighted additive information measures of sum form type, entropies of degree $(\alpha, \beta)$. 1980 AMS SUBJECT CLASSIFICATION CODE. 39B30, $94 \mathrm{~A} 17$.

1. INTRODUCTION

$$
\text { Let } \Gamma_{k}=\left\{\mathrm{p}=\left(\mathrm{p}_{1}, \ldots, \mathrm{p}_{\mathrm{k}}\right): \mathrm{p}_{\mathrm{i}} \geqslant 0, \sum_{i=1}^{\mathrm{k}} \mathrm{p}_{\mathrm{i}}=1\right\}, \mathrm{k} \geqslant 2 .
$$

We say that an information measure $I_{k}: \Gamma_{k} \rightarrow \mathbf{R}, k \geqslant 2$ is $(n, m)$-weighted additive $(n, m \varepsilon \mathbb{N})$ if there exist weight functions $\mathrm{G}_{\mathrm{k}}, \mathrm{L}_{\mathrm{k}}: \Gamma_{\mathrm{k}} \rightarrow \mathbf{R}, \mathrm{k} \geqslant 2$ such that

$$
I_{n m}(P \cdot Q)=G_{n}(P) I_{m}(Q)+I_{n}(P) L_{m}(Q), P \varepsilon \Gamma_{n}, Q \varepsilon \Gamma_{m}
$$

where as usual $\mathrm{P} \cdot \mathrm{Q}=\left(\mathrm{p}_{1} \mathrm{q}_{1}, \ldots, \mathrm{p}_{i} \mathrm{q}_{j}, \ldots, \mathrm{p}_{\mathrm{n}} \mathrm{q}_{\mathrm{m}}\right) \varepsilon \Gamma_{\mathrm{nm}}$. If in addition $I_{k}, G_{k}, L_{k}$ have the sum property with generating functions $I, G, L:[0,1] \rightarrow \mathbf{R}$, that is 


$$
I_{k}(P)=\sum_{i=1}^{k} I\left(p_{i}\right), G_{k}(P)=\sum_{i=1}^{k} G\left(p_{i}\right), L_{k}(P)=\sum_{i=1}^{k} L\left(p_{i}\right)
$$

then equation ( 1.1$)$ goes over into the functional equation

$$
\sum_{i=1}^{n} \sum_{j=1}^{m} I\left(p_{i} q_{j}\right)=\sum_{i=1}^{n} \sum_{j=1}^{m} G\left(p_{i}\right) I\left(q_{j}\right)+\sum_{i=1}^{n} \sum_{j=1}^{m} L\left(q_{j}\right) I\left(p_{i}\right) \text {, }
$$

$P \varepsilon \Gamma_{n}, Q \varepsilon \Gamma_{m}$. This functional equation (1.3) is of interest since the special cases

$$
G(p)=p, L(p)=p+\lambda I(p) \quad, \lambda \varepsilon R
$$

and

$$
G(p)=p^{\alpha}, L(p)=p^{\beta} \quad, \alpha, \beta \in R
$$

play important roles in the characterization of the entropies of degree a (Losonczi, [1])

$$
I_{k}^{a}(P)=\left\{\begin{array}{cl}
H_{k}^{(a, 1)}(P)=\left(2^{1-a}-1\right)^{-1} \sum_{i=1}^{k}\left(p_{i}^{a}-p_{i}\right) & a \neq 1 \\
H_{k}^{1}(P)=-\sum_{i=1}^{k} p_{i} \log p_{i} & a=1
\end{array} \quad P \varepsilon \Gamma_{k}\right.
$$

and degree $(\alpha, \beta)$ (Sharma and Taneja, [2])

$I_{k}^{(\alpha, \beta)}(P)=\left\{\begin{array}{cll}H_{k}^{(\alpha, \beta)}(P)=\left(2^{1-\alpha}-2^{1-\beta}\right)^{-1} \sum_{i=1}^{k}\left(p_{i}^{\alpha}-p_{i}^{\beta}\right) & \alpha \neq \beta & \\ H_{k}^{\alpha}(P)=-2^{\alpha-1} \sum_{i=1}^{k} p_{i}^{\alpha} \log p_{i} & \alpha=\beta\end{array} \quad P \varepsilon k_{k^{\prime}}(1.7)\right.$ respectively. Here we follow the conventions

$$
\log =\log _{2}, 0 \cdot \log 0=0 \text { and } O^{a}=0, a \varepsilon R \text {. }
$$

The aim of this paper is to determine all measurable triples $(I, G, L)$ satisfying (1.3) for a fixed pair $(n, m), n \geqslant 3, m \geqslant 3$ where - because of the known results and the convention (1.8) we assume

$$
G(0)=L(0)=0, G(1)=L(1)=1 .
$$

Thus we determine not only all measurable functions $I$ of $\left(I_{k}\right)$ (see (1.2)) but also all possible choices for $G$ and $L$ in (1.3). Therefore the results due to Kannappan [3-5], Losonczi [1], Sharma and Taneja $[2,6]$ are special cases of our main result. Moreover, if we assume that $I$ is not constant and that $G$ and $L$ are continuous then we can interpret our result in the form that, without loss of generality, we may assume that $G$ and $L$ in (1.3) are continuous, non zero multiplicative functions, that is they are non zero continuous solutions of the functional equation

$$
M(p \cdot q)=M(p) \cdot M(q) \quad p, q \varepsilon[0,1] .
$$

2. MAIN RESULTS.

We make use of the following well known result (Kannappan, [5]). LEMMA 1. Let $n \geqslant 3$ be a fixed integer and let $F:[0,1] \rightarrow \mathbb{R}$ be a measurable function satisfying 


$$
\sum_{i=1}^{n} F\left(p_{i}\right)=0
$$

for all $P \& \Gamma_{n}$. Then there exists a constant a such that

$$
F(p)=a(1-n p), \quad p \varepsilon[0,1] \text {. }
$$

Now we are ready to prove our main result which is an extension of the results, mentioned above.

THEOREM 2. Let $I, G, L:[0,1] \rightarrow \mathbf{R}$ be measurable and let $I$ be non constant. Then $I, G, L$ satisfy $(1.9)$ and (1.3) for a fixed pair $(n, m), n>3, m \geqslant 3$ if, and only if they are of one of the following forms :

$I(p)=a\left(p^{A}-p^{B}\right)$,

$G(p)=(1-b) p^{A}+b p^{B}, L(p)=b p^{A}+(1-b) p^{B}, A \neq B$,

$I(p)=a p^{A} \log p$,

$G(p)=p^{A}(1+b \log p), L(p)=p^{A}(1-b \log p), A \neq 1$,

$I(p)=I(O)+(m n-m-n) I(0) p+d p l o g p, G(p)=L(p)=p$,

$I(1)=0, G(1)=1$

$I(p)=a p^{A}, G(p)=(1-b) p^{A}, L(p)=b p^{A}, p \in[0,1)$

$I(p)=a^{A} \sin (c \log p), G(p)=p^{A}[\cos (c \log p)+b \sin (c \log p)]$,

$$
L(p)=p^{A}[\cos (c \operatorname{cog} p)-b \operatorname{bin}(c \log p)] \text {. }
$$

Here $A, B, a, b, c, d$ are constants and we follow the conventions

$$
O^{a} \cdot \cos (\log O)=0, O^{a} \cdot \sin (\log O)=0, a \in R \text {. }
$$

PROOF. Obviously, the solutions $(I, G, L)$ given by $(2.1)$ to

(2.5) satisfy (1.9) and (1.3). To prove the converse let us introduce the function $I^{\prime}:[0,1] \rightarrow \mathbf{R}$ defined by

$$
I^{\prime}(p)=I(p)-I(0)-(I(1)-I(0)) p, p \varepsilon[0,1] \text {. }
$$

It is clear that I' fulfills

$$
I^{\prime}(0)=I^{\prime}(1)=0 \text {. }
$$

We now show that the triple $\left(I^{\prime}, G, L\right)$ also satisfies (1.3). To see this let us put $P=(1,0,0, \ldots, 0) \varepsilon \Gamma_{n}$ and $Q=(1,0,0, \ldots, 0) \varepsilon \Gamma_{m}$ into (1.3). Using (1.9) we arrive at

$$
I(1)+(n m-1) I(0)=I(1)+(m-1) I(0)+I(1)+(n-1) I(0)
$$

or

$$
I(1)-I(0)=(m n-m-n) I(0) \text {. }
$$

Thus I' can also be written in the form

$$
I^{\prime}(\mathrm{p})=I(\mathrm{p})-I(0)-(m n-m-n) I(0) p .
$$

Substituting $P \in \Gamma_{n^{\prime}} Q=(1,0,0, \ldots, 0) \varepsilon \Gamma_{m}$ and $P=(1,0,0, \ldots, 0) \varepsilon \Gamma_{n}$, $Q \in \Gamma_{m}$ separately into (1.3) we get

$$
\sum_{i=1}^{n} G\left(p_{i}\right)(I(1)+(m-1) I(0))=(n m-n) I(0)
$$

and 


$$
\sum_{j=1}^{m} L\left(q_{j}\right)(I(1)+(n-1) I(0))=(n m-m) I(0)
$$

or, using (2.8)

and

$$
\left(1-\sum_{j=1}^{n} G\left(p_{i}\right)\right)(n m-n) I(0)=0
$$

$$
\left(1-\sum_{j=1}^{m} L\left(q_{j}\right)\right)(n m-m) I(0)=0,
$$

respectively.

After these preparations we can see immediately that I',G,L satisfy

$\sum_{i=1}^{n} \sum_{j=1}^{m} I^{\prime}\left(p_{i} q_{j}\right)=\sum_{i=1}^{n} \sum_{j=1}^{m} G\left(p_{i}\right) I^{\prime}\left(q_{j}\right)+\sum_{i=1}^{n} \sum_{j=1}^{m} L\left(q_{j}\right)^{\prime}\left(p_{i}\right)$

for all $\mathrm{P} \varepsilon \Gamma_{\mathrm{n}}, \mathrm{Q} \varepsilon \Gamma_{\mathrm{m}}$. Putting $I^{\prime}$, given by $(2.6)$, into $(2.14)$ and using (1.3) and (2.8), we see that (2.14) is equivalent to

$$
\left(1-\sum_{i=1}^{n} G\left(p_{i}\right)\right)(n m-n) I(0)+\left(1-\sum_{j=1}^{m} L\left(q_{j}\right)\right)(n m-m) I(0)=0 .(2.15)
$$

But (2.15) is indeed valid because of (2.12) and (2.13).

In a further step we derive a functional equation for I', G and $L$ in which no sums will occur. Setting

$$
F(p, q)=I^{\prime}(p \cdot q)-G(p) I^{\prime}(q)-L(q) I^{\prime}(p), p, q \varepsilon[0,1]
$$

we get from $(2.14)$

$$
\sum_{i=1}^{n} \sum_{j=1}^{m} F\left(p_{i}, q_{j}\right)=0, P \varepsilon \Gamma_{n}, Q \varepsilon \Gamma_{m} \text {. }
$$

Since by hypothesis $F:[0,1]^{2} \rightarrow \mathbf{R}$ is measurable in each variable we get from Lemma 1 in Kannappan [3] (This Lemma is an application of the above Lemma 1) that $F$ can be represented in the form

$$
\begin{aligned}
F(p, q) & =F(p, 0)(1-m q)+F(0, q)(1-n p)- \\
& -F(0,0)(1-m q)(1-n p) .
\end{aligned}
$$

Thus (2.16) and (2.17) imply

$$
I^{\prime}(p \cdot q)=G(p) I^{\prime}(q)+L(q) I^{\prime}(p), p, q \varepsilon[0,1]
$$

since $(2.17),(1.9)$ and $(2.7)$ yield $F(p, 0)=F(0, q)=F(0,0)=0$. Because of $I^{\prime}(0)=G(0)=L(0)=0$ it is enough to solve (2.18) for all $\mathrm{p}, \mathrm{q} \varepsilon(0,1]$. Complex-valued functional equations of this type were intensively studied by Vincze [7-9]. From these results we get the solutions of $(2.18)$ for $p, q \in(0,1]$ (Ebanks, [10]) which have one of the following forms : 


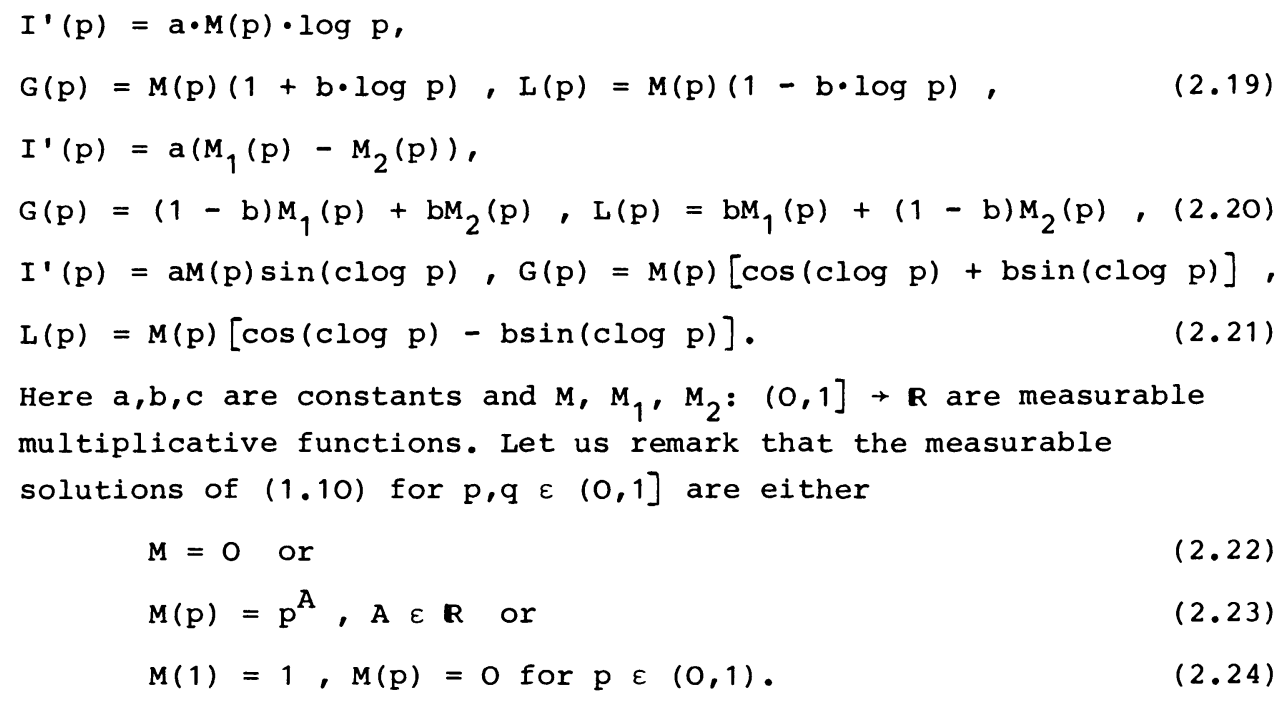

Since I has the form

$$
I(p)=I^{\prime}(p)+I(0)+(n m-n-m) I(0) p, p \varepsilon[0,1]
$$

(see $(2.6)-(2.8)$ ) we can derive the solutions $(I, G, L)$ of $(1.9)$ and (1.3) from (2.19) to $(2.24)$. Let us first consider the case that

$$
\sum_{i=1}^{n}\left(G\left(p_{i}\right)-p_{i}\right)=0 \text { and } \sum_{j=1}^{m}\left(L\left(q_{j}\right)-q_{j}\right)=0
$$

for all $P \in \Gamma_{n}, Q \in \Gamma_{m}$. Then Lemma 1 implies that

$$
G(p)=r p+s, L(p)=r^{\prime} p+s^{\prime}, p \varepsilon[0,1]
$$

where $r, r^{\prime}, s^{\prime} s^{\prime}$ are constants. Because of (1.9) we arrive at

$$
G(p)=L(p)=p, p \in[0,1] \text {. }
$$

This is only possible if either

$$
\mathrm{b}=0, M(\mathrm{p})=\mathrm{p} \text { in }(2.19)
$$

or if

$$
b=1, M_{1}(p)=M_{2}(p)=p \text { in }(2.20) .
$$

In both cases we get the solution (2.3). Now we assume that

$$
\begin{aligned}
& \sum_{i=1}^{n}\left(G\left(p_{i}\right)-p_{i}\right) \neq 0 \text { for some } P \in \Gamma_{n} \text { or } \\
& \sum_{j=1}^{m}\left(L\left(q_{j}\right)-q_{j}\right) \neq 0 \text { for some } Q \varepsilon \Gamma_{m} \cdot
\end{aligned}
$$

Then (2.12) and (2.13) imply that $I(0)=0$ so that in all cases where

$$
G(p) \neq p \quad \text { or } \quad L(p) \neq p
$$


we get that $I$ is equal to $I^{\prime}$ and thus $I$ is not dependent upon $n$ and $m$ (see (2.25)). Using $G(1)=L(1)=1$ and the hypothesis that $I$ is not constant we obtain from $(2.19)$ to $(2.24)$ the remaining solutions $(2.1),(2.2),(2.4)$ and (2.5). Thus the Theorem is proven. It is clear that we can obtain from Theorem 2 some new characterization theorems for information measures. For instance, we remark that the functions $G$ and $L$ given by (2.4) or (2.5) cannot be continuous simultaneously. Thus we get the following extension of results in Kannappan $[3,4]$, Sharma and Taneja $[2,6]$.

COROLLARY 3. If in addition to the hypotheses of Theorem 2, $G$ and $L$ are continuous then the only solutions $(I, G, L)$ of $(1.9)$ and (1.3) are given by $(2.1),(2.2)$ and (2.3) .

corollary 3 implies immediately the following characterization theorem :

Let $I_{k}$ be an $(n, m)$-weighted additive information measure where $I_{k}, G_{k}, L_{k}$ have the sum property with continuous generating functions $I, G, L:[0,1] \rightarrow R$. If

$$
I(O)=G(O)=L(O)=O, G(1)=L(1)=1 \text { and } I\left(\frac{1}{2}\right)=\frac{1}{2}
$$

then $I_{k}(P)=H_{k}^{(A, B)}(P)$ or $I_{k}(P)=H_{k}^{C}(P), P \varepsilon \Gamma_{k}$. Here $A, B, C$ are real constants with $A \neq B$.

Finally we give two interpretations of our result. If we put $\mathrm{b}=0$ into $(2.1),(2.2)$ and (2.3) then we get - with unchanged $I(p)$ -

$$
\begin{array}{ll}
G(p)=p^{A}, L(p)=p^{B}=p^{B}-p^{A}+p^{A}=\frac{1}{a} I(p)+p^{A}, & A \neq B, \\
G(p)=p^{A}, L(p)=p^{A} & , A \neq 1, \\
G(p)=p, L(p)=p, &
\end{array}
$$

respectively. Thus we may consider corollary 3 as a justification for the fact that in the literature only two special forms of $G$ and L were considered, namely (1.4) and (1.5).

On the other hand, the condition $b=0$ in (2.1) and (2.2) implies that in Corollary 3 we may assume without loss of generality that $G$ and $L$ are continuous, non zero multiplicative functions. This result is analogous to a result concerning recursive measures of multiplicative type (Aczél and $\mathrm{Ng},[11]$ ).

\section{REFERENCES}

1. LOSONCZI, L. A characterization of entropies of degree $\alpha$, Metrika 28 (1981), 237-244.

2. SHARMA, B.D. and TANEJA, I.J. Entropy of type $(\alpha, \beta)$ and other generalized measures of information theory, Metrika 22 (1975), 205-215.

3. KANNAPPAN, $P 1$ 。 On some functional equations from additive and non-additive measures-I, Proc. Edin. Math. Soc. 23 (1980), 145-150. 
4. KANNAPPAN, Pl. On a generalization of sum form functional equation-III, Demonstratio Math. 13 (1980), 749-754.

5. KANNAPPAN, Pl. On a generalization of sum form functional equation-I, Stochastica 2 (1983), 97-109.

6. SHARMA, B.D. and TANEJA, I.J. Three generalized additive measures of entropy, EIK 13(7/8) (1977), 419-433.

7. VINCZE, E. Uber die Verallgemeinerung der trigonometrischen und verwandten Funktionalgleichungen, Ann. Univ. Sci. Budapest Eötvös, Sect. Math. 3-4 (1960-1961), 389-404.

8. Vincze, E. Eine allgemeinere Methode in der Theorie der Funktionalgleichungen II, Publ. Math. Debrecen 9 (1962), 314-323.

9. Vincze, E. Eine allgemeinere Methode in der Theorie der Funktionalgleichungen III, Publ. Math. Debrecen 10 (1963), 191-202.

10. Ebanks, B.R. On measures of fuzziness and their representations, J. Math. Anal. Appl. 94 (1983), 24-37.

11. ACZEL, J. and NG, C.T. Determination of all semisymmetric recursive information measures of multiplicative type on $n$ positive discrete probability distributions, Linear Algebra Appl. 52/53 (1983), 1-30. 


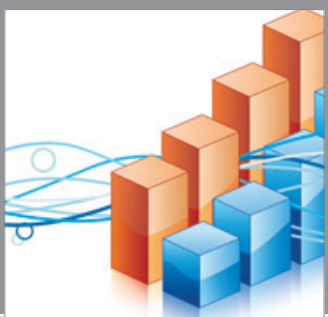

Advances in

Operations Research

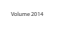

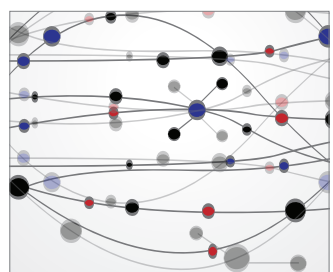

\section{The Scientific} World Journal
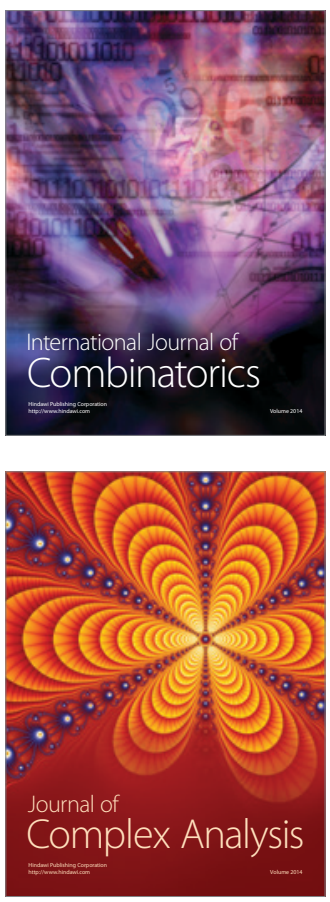

International Journal of

Mathematics and

Mathematical

Sciences
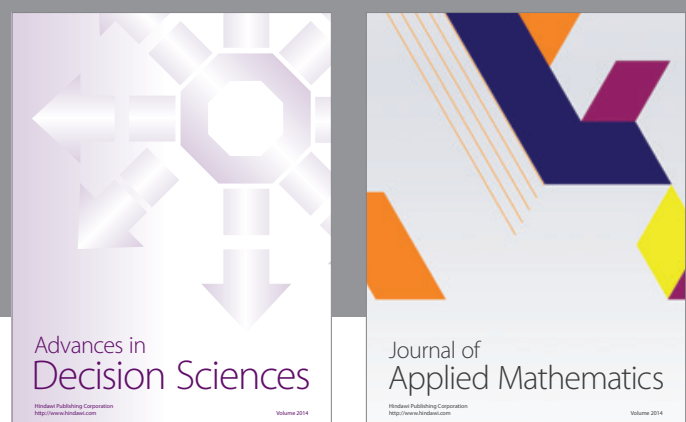

Journal of

Applied Mathematics
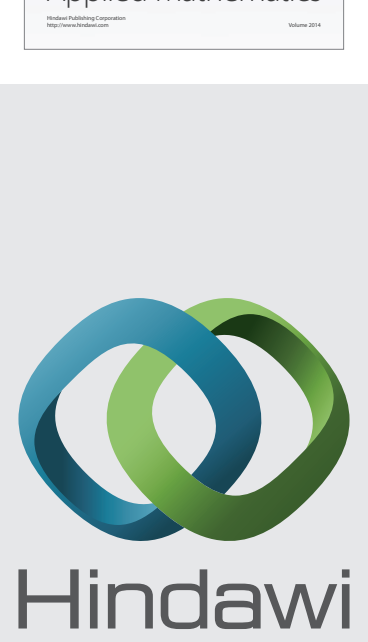

Submit your manuscripts at http://www.hindawi.com
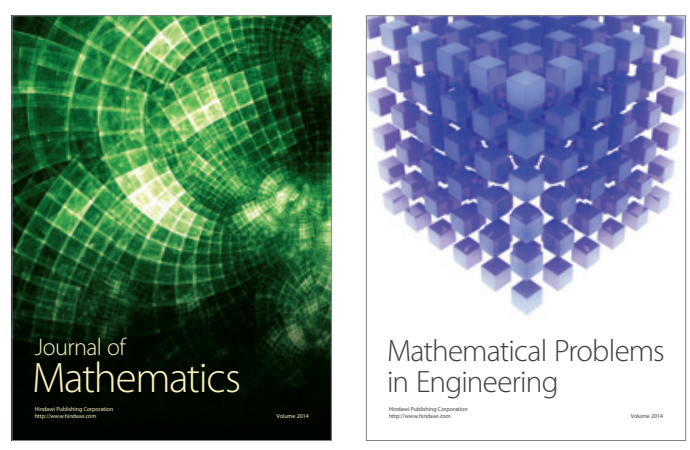

Mathematical Problems in Engineering
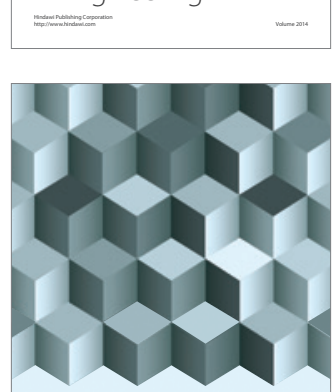

Journal of

Function Spaces
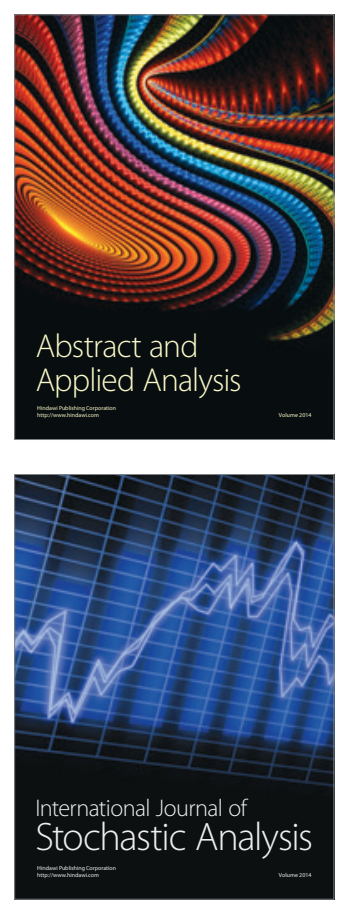

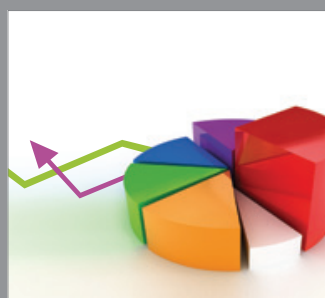

ournal of

Probability and Statistics

Promensencen
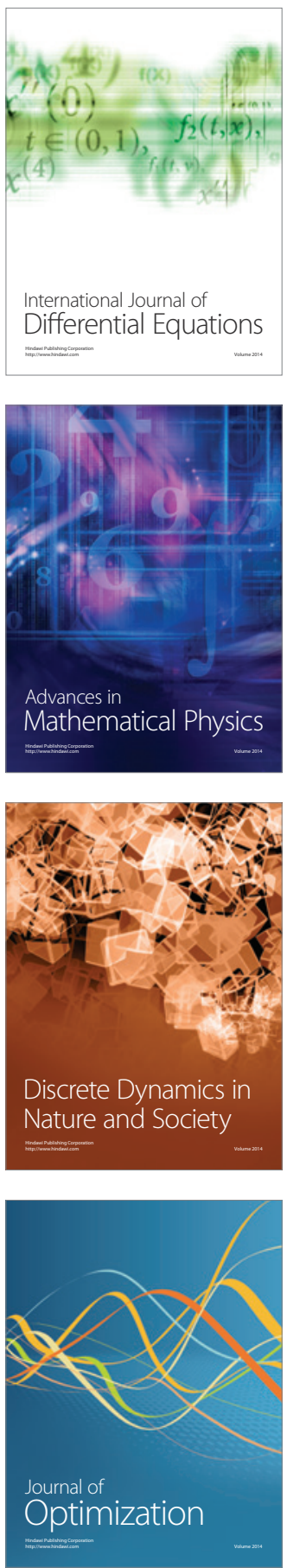